\title{
Caracterización morfológica de cobre depositado por electrólisis influenciado por presencia de iones cloruros
}

\author{
Morphological characterization of copper \\ deposited by electrolysis influenced by \\ presence of chloride ions
}

Alvaro Soliz ${ }^{1}$, Catalina Alfaro ${ }^{1}$, Luis Cáceres ${ }^{2}$, Danny Guzman ${ }^{1}$

\author{
${ }^{1}$ Departamento de Ingeniería en Metalurgia, Universidad de Atacama, Av. Copayapu 485, Copiapó, Chile \\ ${ }^{2}$ Departamento de Ingeniería Química y Procesos de Minerales, Universidad de Antofagasta, Av. Angamos 601, Antofa- \\ gasta, Chile \\ e-mail: alvaro.soliz@uda.cl
}

\section{RESUMEN}

En el presente trabajo se estudia el efecto de la densidad de corriente sobre la morfología del cobre depositado por electrólisis desde una solución ácida de sulfato de cobre en presencia y ausencia de iones cloruros como impurezas. Para ello, técnicas de cronopotenciometría y microscopía electrónica de barrido son utilizadas. El estudio revela que, para ambas situaciones en presencia y ausencia de iones cloruros, los perfiles temporales del potencial catódico son desplazados en dirección catódica con el incremento de la densidad de corriente, los cuales están asociados a la ocurrencia simultánea de reacciones catódicas durante la electrólisis de cobre. Los resultados morfológicos demuestran una influencia directa de la densidad de corriente representada por transiciones en los mecanismos de electrocristalización asociadas a etapas de nucleación y crecimiento de las partículas depositadas. Los cambios en los patrones morfológicos son corroborados por un depósito de cobre tipo dendrítico y granular en la solución de electrolitos sin iones cloruros, a diferencia del depósito de cobre observado en la solución de electrolitos con presencia de iones cloruros los cuales se observa una morfología dendrítica y laminada. Estos resultados claramente demuestran aspectos mecanísticos sobre la electrólisis de cobre.

Palabras clave: Densidad de corriente, Iones cloruros, Morfología, Potencial catódico.

\begin{abstract}
In the present work, the effect of current density on the morphology of copper deposited by electrolysis from an acidic solution of copper sulfate in the presence and absence of chloride ions as impurities is studied. For this purpose, chronopotentiometry and scanning electron microscopy techniques are used. The study reveals that, for both situations, in the presence and absence of chloride ions, the time profiles of the cathodic potential are shifted in cathodic direction with the increase of the current density. These time profiles are associated with the simultaneous occurrence of cathodic reactions during copper electrolysis. The morphological results demonstrate a direct influence of the current density represented by transitions in the electrocrystallization mechanisms associated with nucleation and growth stages of the deposited particles. Changes in the morphological patterns are corroborated by a dendritic and granular type copper deposit in the electrolyte solution without chloride ions, in contrast to the copper deposit observed in the electrolyte solution with the presence of chloride ions, where a dendritic and laminar morphology is observed. These results clearly demonstrate the mechanistic aspects on copper electrolysis.
\end{abstract}

Keywords: Current density, Chloride ions, Morphology, Cathodic potential.

\section{INTRODUCCION}

El cobre es uno metales de mayor demanda a nivel industrial debido a sus excelentes propiedades de conductividad térmica, conductividad eléctrica, propiedades catalíticas, ductilidad, maleabilidad, las cuales son de 
amplio aprovechamiento en el desarrollo de múltiples aplicaciones como intercambiadores de calor, contactores eléctricos, electroimanes, aleante de nuevos materiales, entre otros. Actualmente, una de las vías más utilizadas para su obtención a escala industrial es a través del proceso de electrólisis, donde el metal es obtenido por un proceso electroquímico donde la reducción de iones cúpricos presentes en una solución acuosa ácida toma lugar (reacción 1) [1, 2]. La naturaleza electroquímica de este proceso tiene como contraparte la oxidación de moléculas de agua (reacción 2), la cual ocurre sobre ánodos típicamente fabricados por una aleación de $\mathrm{Pb}-\mathrm{Ca}-\mathrm{Sn}$.

$$
\begin{aligned}
& \mathrm{Cu}^{+2}+2 \mathrm{e}^{-} \rightarrow \mathrm{Cu} \\
& \mathrm{H}_{2} \mathrm{O} \rightarrow 1 / 2 \mathrm{O}_{2}+2 \mathrm{H}^{+}+2 \mathrm{e}^{-}
\end{aligned}
$$

Este método de obtención electrolítica está caracterizada por el manejo de parámetros cinéticos y termodinámicos expresados en términos de densidad de corriente y potencial de celda [3-5], los cuales dependen fuertemente de las condiciones del tipo de solución de electrolitos y la presencia de posibles contaminantes, tales como iones y/o especies de $\mathrm{Fe}, \mathrm{Cl}$, As, $\mathrm{Pb}$ u otras, los que provienen desde etapas metalúrgicas previas como la lixiviación y/o extracción por solventes. Como resultado de este proceso, el depósito de cobre catódico obtenido y la presencia de contaminantes constituyen un factor crítico en cuanto a la calidad física y química de este metal. Típicamente, un exceso en la concentración de iones cloruros (>50 ppm) en la solución de electrolitos afecta principalmente la apariencia del depósito promoviendo un cobre metálico de carácter rugoso, opaco y dendrítico, en adición a otros efectos tales como la formación de picaduras sobre la superficie de los cátodos de acero inoxidable debido a un proceso de corrosión, y la adsorción de cloruros de cobre insolubles sobre los ánodos dificultando de esta forma el proceso de electrólisis. Diversos estudios se enfocaron al estudio de oxidación del agua sobre electrodos anódicos [6-9], y otros estudios evaluaron la morfología del depósito metálico influenciado por parámetros eléctricos y físico-químicos [10-18]. La mayoría de los estudios revela que pueden obtenerse tamaños de partícula micrométricas hasta nanométricas mediante cambios en la densidad de corriente [19-22]. Sin embargo pocos trabajos consideran soluciones de electrolitos con presencia de elevados niveles de iones cloruros, y cómo afecta su respuesta electroquímica y morfológica del proceso de electrólisis. CHRZANOWSKA y MROCZKA [15] encontraron que la mejor uniformidad en la rugosidad del cobre electrodepositado se obtuvo a concentraciones de $7.618 \times 10^{-8} \mathrm{~mol} / \mathrm{L}$. Por otra parte, MROCZKA et al. [23] reportaron que la presencia de cloruros en la solución de electrolitos promueve un cobre electrodepositado muy rugoso y corroído con numerosos sitios de picadura, debido a una corrosión acelerada, en comparación con soluciones de electrolitos conteniendo aditivos como el polietilenglicol. SHAO et al. [24] en sus estudios sobre la influencia de los iones cloruros en la electrolisis de cobre revelaron que una alta concentración de éstos iones promueve la formación de complejos clorurados de cobre, reduciendo de esta forma la concentración de iones cúpricos libres en la solución e induciendo una polarización catódica del proceso de electrólisis.

En este trabajo se reporta el estudio morfológico del cobre depositado, vía un proceso de electrolisis, y como éste es influenciado por la presencia de altos niveles de iones cloruros. Este enfoque se basa en la variabilidad del potencial de electrodo en respuesta a cambios en la densidad de corriente. La influencia de los iones cloruros fueron correlacionados con aspectos morfológicos y patrones temporales en los potenciales de electrodo.

\section{MATERIALES Y MÉTODOS}

Los ensayos fueron realizados utilizando una celda electroquímica convencional de tres electrodos. Los electrodos de trabajo fueron fabricados desde barras comerciales de acero inoxidable AISI 316L, los cuales fueron moldeados cilíndricamente, y selladas dentro una vaina de teflón con resina epóxica garantizando de esta forma que solo la sección circular (transversal), de área igual a $63.62 \mathrm{~mm}^{2}$, esté expuesta a la solución de electrolitos. Se utilizó un electrodo de $\mathrm{Ag} / \mathrm{AgCl}$ (sat. $\mathrm{KCl}$ ) y un alambre de platino como electrodos de referencia y auxiliar respectivamente. Previo a los ensayos de electrolisis, los electrodos de trabajo fueron mecánicamente lijados utilizando de forma secuencial papel lija de SiC desde la malla \#600 hasta \#1200, lavados con alcohol isopropílico en un baño ultrasónico, y finalmente enjuagados con agua desmineralizada.

Las soluciones de electrolitos fueron preparadas utilizando reactivos grado analítico y agua desmineralizada. La solución patrón de sulfato de cobre estuvo compuesta por $40 \mathrm{~g} / \mathrm{L} \mathrm{Cu}^{+2}$ y $160 \mathrm{~g} / \mathrm{L} \mathrm{H}_{2} \mathrm{SO}_{4}$, a las que se adicionó $\mathrm{NaCl}$ manteniendo una concentración de 1000 ppm. Todos los ensayos fueron realizados a temperatura ambiente de $24 \pm 0.5^{\circ} \mathrm{C}$, bajo una condición de estancamiento hidrodinámico.

Ensayos cronopotenciométricos de electrólisis de cobre fueron realizados durante 90 min a densidades 
de corriente de 250, 5001000 y $2000 \mathrm{~A} / \mathrm{m}^{2}$ utilizando un potenciostato/galvanostato BioLogic VSP-300, controlado por el software EC-Lab. Todos los potenciales están referidos al electrodo estándar de hidrógeno (EEH).

Posterior a los ensayos de electrolisis, el cobre depositado fue cuidadosamente removido desde la superficie del electrodo de trabajo, para luego ser lavado con abundante agua desmineralizada y acetona en orden a remover trazas de ácido. Finalmente estos productos de la electrólisis fueron puestos dentro un franco plástico con sílica gel y almacenados en una estufa a una temperatura de $60{ }^{\circ} \mathrm{C}$, en orden a limitar una futura oxidación, para su futura caracterización morfológica.

La caracterización morfológica del cobre depositado fue observada utilizando un microscopio electrónico de barrio Zeiss EVO MA10 SEM (Scanning Electron Microscope).

\section{RESULTADOS Y DISCUSION}

\subsection{Mediciones cronopotenciométricas}

La Figura 1 muestra perfiles temporales del potencial de electrodo medidos durante el proceso de electrólisis de cobre a diferentes densidades de corriente, utilizando una solución de electrolitos con ausencia de iones cloruros. Desde estos resultados, es posible observar que para una densidad de corriente de $250 \mathrm{~A} / \mathrm{m}^{2}$, las cuales son típicamente utilizadas a nivel industrial para la electrólisis de cobre, los perfiles del potencial de electrodo revelan una tendencia suavizada y constante durante todo el transcurso de electrólisis, alcanzando un valor estacionario de $76 \mathrm{mV} / \mathrm{EEH}$. Por otra parte, para densidad de corriente mayores a $250 \mathrm{~A} / \mathrm{m}^{2}$, las curvas de potencial de electrodo revelan formas similares y simétricas donde las magnitudes numéricas son desplazadas catódicamente con el incremento en la densidad de corriente, la cual es observada durante todo el transcurso del proceso de electrólisis. Acorde a estos resultados, es posible indicar que la electrólisis inicia con potenciales altamente catódicos alcanzando valores de $-489,-550$ y $-752 \mathrm{mV} / \mathrm{EEH}$ para densidades de corriente de 500, 1000 y $2000 \mathrm{~A} / \mathrm{m}^{2}$ respectivamente. Posteriormente, la variabilidad temporal de este potencial revela una tendencia hacia valores más anódicos durante los primeros 20 min donde se observan múltiples perturbaciones en la respuesta electroquímica, las que futuramente son inhibidas a tiempos superiores que 20 min donde se tienden a alcanzar valores estacionarios e iguales a -152, -174 y -278 mV/EEH para 500, 1000 y $2000 \mathrm{~A} / \mathrm{m}^{2}$ respectivamente.

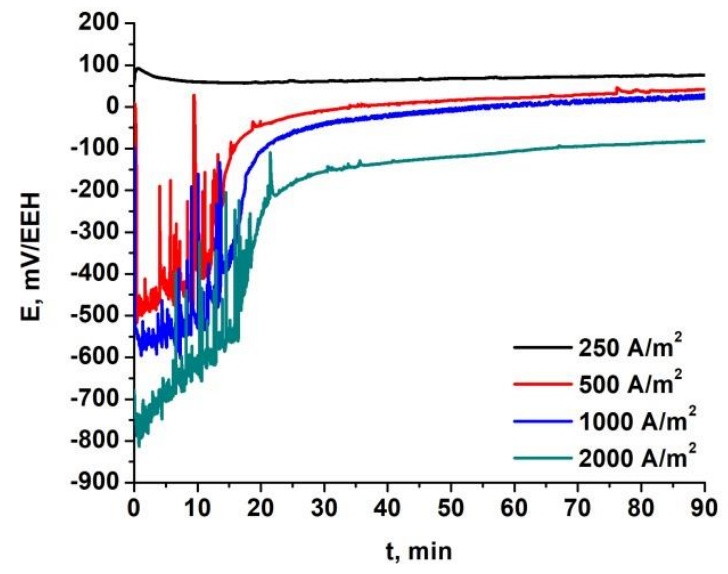

Figura 1: Perfiles temporales del potencial de electrodo para la electrólisis de cobre en solución patrón con ausencia de iones cloruros a diferentes densidades de corriente.

Datos presentados en la Figura 2, evidencian perfiles de potencial de electrodo durante la electrólisis de cobre realizada utilizando la solución patrón con presencia de iones cloruros. En estos resultados se observan patrones temporales similares a los obtenidos durante la electrólisis realizada con ausencia de iones cloruros, caracterizadas por un desplazamiento catódico del potencial de electrodo con el incremento en la densidad de corriente, tanto durante los primeros instantes del proceso como al alcanzar el estado estacionario. Adicionalmente, una comparación directa entre los resultados en presencia y ausencia de iones cloruros, muestran una disminución en la banda temporal asociada a perturbaciones en la respuesta electroquímica la cual fue reducida desde los 20 min hasta los $7 \mathrm{~min}$ en presencia y ausencia de cloruros respectivamente. Estos cam- 
bios claramente revelan una influencia directa de contaminantes como los iones cloruros sobre la electrólisis de cobre.

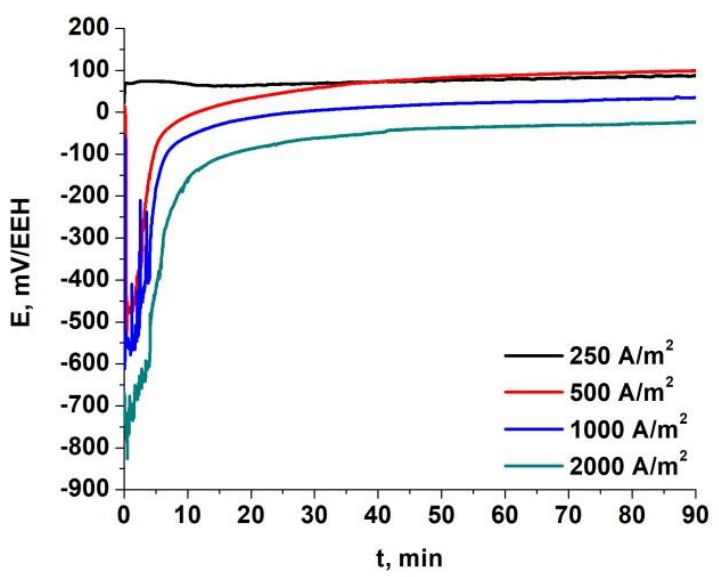

Figura 2: Perfiles temporales del potencial de electrodo para la electrólisis de cobre en solución patrón en presencia de $1000 \mathrm{ppm} \mathrm{NaCl}$ a diferentes densidades de corriente.

La forma y tendencia de estas curvas pueden ser atribuidas a la ocurrencia de reacciones catódicas competitivas sobre la superficie del acero inoxidable promoviendo cambios significativos en la respuesta electroquímica del potencial de electrodo. Desde este punto de vista, y acorde a las características de la solución de electrolitos y los diagramas de Pourbaix para el cobre [25], el desplazamiento catódico de los potenciales de electrodo sería explicado por la presencia de la reacción de evolución de hidrógeno (reacción 3), la cual ocurre paralelamente a la reacción de reducción de cobre (reacción 2):

$$
2 \mathrm{H}^{+}+2 \mathrm{e}^{-} \rightarrow \mathrm{H}_{2}
$$

En este sentido, y acorde a los resultados en las Figuras 1 y 2, es posible indicar que para una densidad de corriente iguale a $250 \mathrm{~A} / \mathrm{m}^{2}$ solo la reacción de reducción de cobre es la que toma lugar sobre el electrodo de trabajo. Sin embargo, a densidades de corrientes iguales y mayores a $500 \mathrm{~A} / \mathrm{m}^{2}$ ambas reacciones de reducción de cobre y evolución de hidrógeno toman lugar sobre el electrodo de trabajo, donde esta última reacción toma mayor preponderancia durante los primeros minutos del proceso de electrólisis, promoviendo de esta forma la precipitación de partículas de cobre con menor tamaño las cuales pueden alcanzar rangos desde micropartículas $(\leq 100 \mu \mathrm{m})$ hasta nanopartículas $(\leq 100 \mathrm{~nm})$. Esta evolución de hidrógeno fue corroborada para ambas soluciones de electrolitos estudiadas, a partir de una observación visual de la abundante generación de microburbujas sobre la superficie del acero inoxidable, las cuales futuramente fueron creciendo por un mecanismo de coalescencia durante todo el rango de tiempo de electrólisis de cobre estudiado. Adicionalmente, las perturbaciones o saltos en el potencial de electrodo y observados dentro los primeros 20 min de electrólisis, pueden estar asociadas a la generación de una turbulencia hidrodinámica debido al desprendimiento de microburbujas de hidrógeno sobre la interface sólido-líquido, promoviendo así un desprendimiento de las partículas de cobre depositadas.

\subsection{Análisis morfológico}

La caracterización morfológica del cobre depositado desde la solución patrón con presencia y ausencia de iones cloruros fue realizada por microscopía electrónica de barrido (SEM). En la Figura 3 se presentan imágenes del cobre depositado desde la solución patrón con ausencia de iones cloruros a diferentes densidades de corriente. Desde la figura, los cambios en la densidad de corriente promueven depósitos de cobre con morfología variable.

Para los ensayos realizados a una densidad de corriente de $250 \mathrm{~A} / \mathrm{m}^{2}$ (Figura 3.a) el depósito revela un crecimiento granular y uniforme con dominios individuales densamente empaquetados que varían entre los 10 y $20 \mu \mathrm{m}$ los que cubren toda el área electroactiva del electrodo de acero inoxidable. Esta característica morfológica en el depósito está en concordancia con la respuesta electroquímica donde la variabilidad temporal en el potencial de electrodo no es afecta a cambios ni saltos abruptos en sus valores. Por otra parte, en relación a los mecanismos presentes durante el proceso de electrocristalización [26], es posible afirmar que el 
mecanismo de crecimiento es predominante durante la electrólisis de cobre llevada a $250 \mathrm{~A} / \mathrm{m}^{2}$.

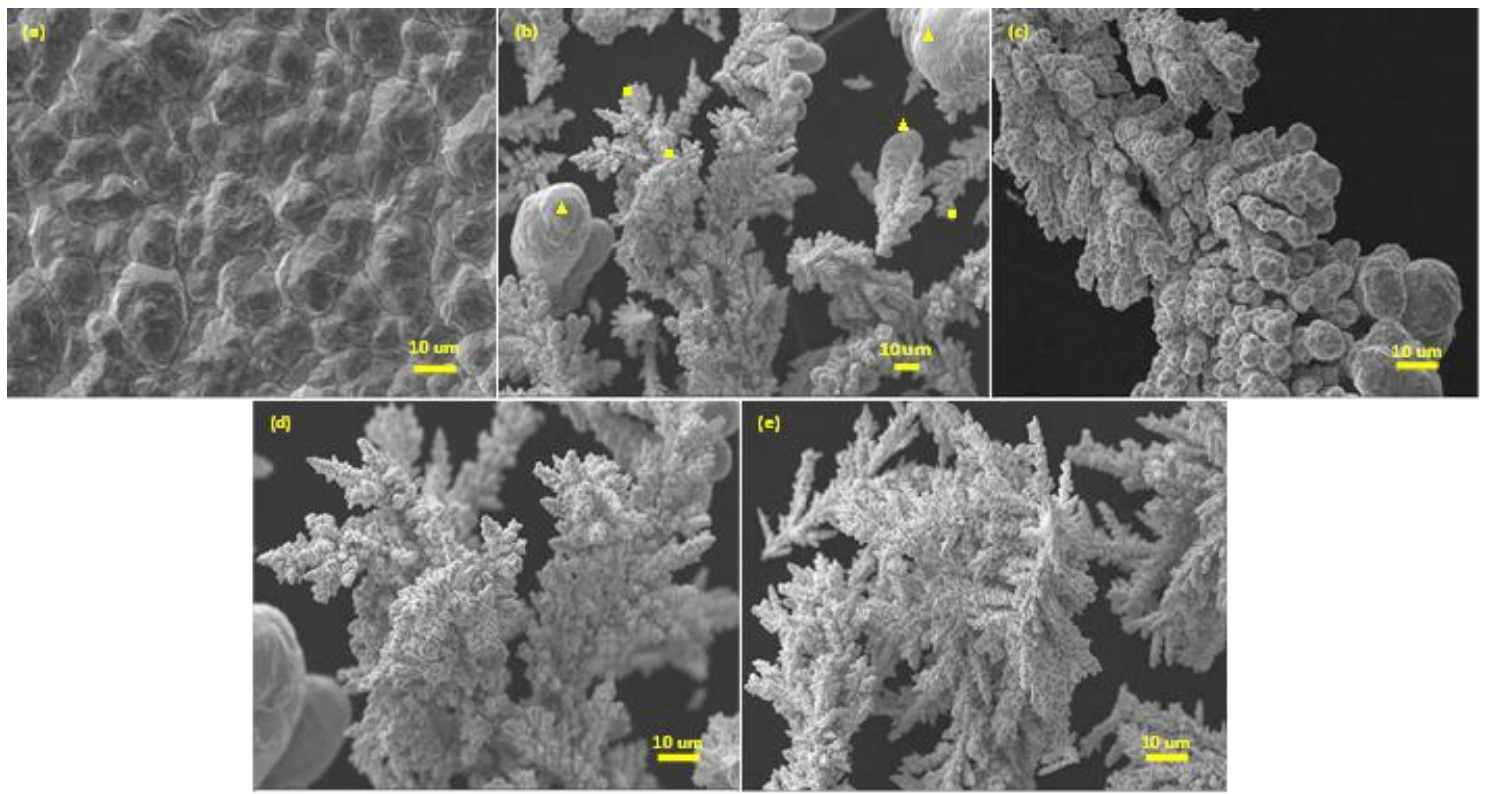

Figura 3: Patrones morfológicos del cobre depositado en ausencia de iones cloruros a densidades de corriente iguales a: a) 250 , b) 1000 , c) 500, d) 1000 y e) $2000 \mathrm{~A} / \mathrm{m}^{2}$.

Adicionalmente, las Figuras 3.b-e muestran cambios significativos en la morfología del depósito de cobre en comparación a la Figura 3.a. Estos cambios claramente pueden ser caracterizados por la presencia de patrones dendríticos y ramificados que poseen partículas granulares de tamaño variable según la densidad de corriente aplicada. Una particularidad observada en todos los ensayos, a diferencia del realizado a $250 \mathrm{~A} / \mathrm{m}^{2}$, es la presentada en a Figura 3.b, donde los patrones morfológicos se caracterizan por la presencia de zonas de alta densidad dendrítica con partículas pequeñas ( $\mathbf{(})$, y zonas con un crecimiento continuo y tamaños de partículas más grandes ( $\boldsymbol{\Delta})$ (Figura 3.b).

Para densidades de corriente de $500 \mathrm{~A} / \mathrm{m}^{2}$ (Figura 3.c), el depósito de cobre muestra formas granulares con tamaños de partícula promedio de $3 \mu \mathrm{m}$ en la zona de alta densidad dendrítica y $10 \mu \mathrm{m}$ en las zonas de crecimiento continuo. Con el incremento en la densidad de corriente, los patrones dendríticos (Figura 3.d y Figura 3.e) revelan una ligera disminución en el tamaño de partícula las que varían desde los $2 \mu \mathrm{m}$ hasta un tamaño de $1.3 \mu \mathrm{m}$ para 1000 y $2000 \mathrm{~A} / \mathrm{m}^{2}$ respectivamente.

En base a la caracterización morfológica y la variabilidad temporal del potencial de electrodo, es posible indicar que en una solución de electrolitos con ausencia de iones cloruros, la forma granular del cobre depositado es independiente de la densidad de corriente aplicada. Sin embargo, el efecto de esta variable es apreciable sobre los mecanismos de electrocristalización, donde el mecanismo de nucleación de partículas es la preponderante a densidades de corriente mayores o iguales a $500 \mathrm{~A} / \mathrm{m}^{2}$, la cual también además es afecta por la reacción de evolución de hidrogeno quien promueve una disminución en zonas activas del electrodo de trabajo donde ocurre la reducción del cobre. Adicionalmente, estos cambios en la morfología dendrítica y la limitación de su crecimiento pueden explicar las perturbaciones observadas en los potenciales de electrodo.

La Figura 4 muestra la caracterización morfológica del depósito de cobre obtenida desde la solución patrón con un contenido de $1000 \mathrm{ppm}$ de $\mathrm{NaCl}$, a diferentes densidades de corriente. Desde esta figura, una apreciación inicial indica la influencia de los iones cloruros sobre la morfología del depósito de cobre. Para visualizar estos cambios, el depósito de cobre obtenido a $250 \mathrm{~A} / \mathrm{m}^{2}$ revela una morfología compacta observada a nivel macroscópico, sin embargo a nivel microscópico (Figura 4.a) la morfología del depósito claramente muestra dominios más separados de estructura laminar y forma triangular, los cuales alcanzan tamaños de partícula menores a $10 \mu \mathrm{m}$. Si bien a esta densidad de corriente, la variabilidad temporal del potencial de electrodo no revela cambios sustanciales en su tendencia (Figura 2), una inspección microscópica del cobre depositado revela claramente el efecto de los iones cloruros sobre ésta. 


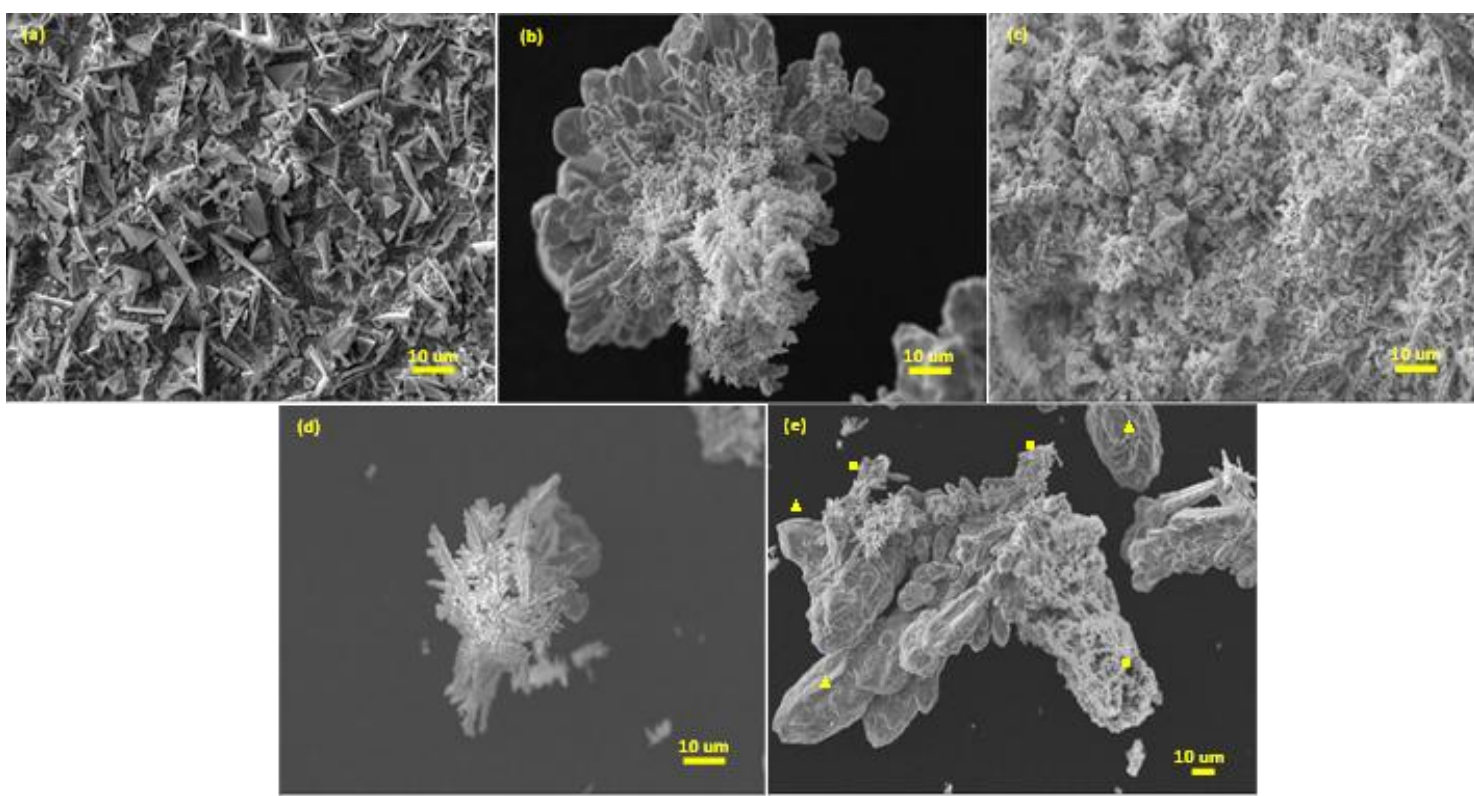

Figura 4: Patrones morfológicos del cobre depositado en presencia de $1000 \mathrm{ppm} \mathrm{NaCl}$ a densidades de corriente iguales a: a) 250 , b) 500 , c) 1000 , d) 2000 y e) $2000 \mathrm{~A} / \mathrm{m}^{2}$.

Para densidades de corriente de $500 \mathrm{~A} / \mathrm{m}^{2}$ y superiores (Figura 4.b-e), los patrones morfológicos revelan características similares a una estructura dendrítica, donde también se observan zonas de partículas pequeñas (๘) y zonas de crecimiento continuo ( $\mathbf{\Lambda}$ ) (Figura 4.e). Adicionalmente, es visto que los patrones dentro la región dendrítica aún poseen dominios laminados, a diferencia de la zona de crecimiento continuo donde se observan dominios granulares. Por otra parte, estos resultados muestran que incrementando la densidad de corriente se enlaza a una disminución en el tamaño de partículas de cobre dentro la matriz dendrítica, las cuales poseen un tamaño promedio de $1.5,0.9$ y $0.4 \mu \mathrm{m}$ para 500,1000 y $2000 \mathrm{~A} / \mathrm{m}^{2}$ respectivamente. A medida que el proceso de electrólisis avanza, el cobre depositado muestra una reducción sustancial de las zonas dendríticas, promoviéndose una mayor densidad en las zonas de crecimiento continuo de las partículas de cobre (Figura 4.e). Este incremento en las zonas de crecimiento continuo puede ser explicado en términos de la variabilidad en los potenciales de electrodo (Figura 2), donde la banda temporal asociada a la formación de micropartículas es más estrecha.

\section{CONCLUSIONES}

En el presente trabajo se estudió el proceso de electrólisis de cobre influenciado por la presencia de iones cloruros como contamines por mediciones cronopotenciométricas e inspecciones morfológicas. Las mediciones electroquímicas exhiben una clara dependencia de la densidad de corriente sobre el potencial catódico, tanto en presencia como ausencia de iones cloruros en la solución de electrolitos. Una combinación entre factores asociados a mecanismos de electroprecipitación por nucleación y crecimiento explica los cambios en patrones morfológicos revelando una densidad de corriente límite la cual determina un depósito de cobre compacto y homogéneo y un depósito dendrítico con tamaños de partícula variables. La presencia de iones cloruros en la solución de electrolitos fue un factor relevante tanto en los cambios temporales del potencial electrodo catódico como en los patrones morfológicos del cobre depositado. Estos efectos fueron evidenciados por cambios en la estructura y forma geométrica de los dominios individuales de cobre, los que además fueron influenciados por la densidad de corriente aplicada.

\section{AGRADECIMIENTOS}

Los autores agradecen el apoyo financiero del Fondo de Innovación para la Competitividad-Región de Atacama FIC-40013608-0, llevado por el Departamento de Ingeniería en Metalurgia de la Universidad de Atacama.

\section{BIBLIOGRAFÍA}

[1] HABASHI, F., Handbook of extractive metallurgy, 1ª ed., Weinheim, Wiley-VCH, 1997. 
[2] POPOV, K.I., DJOKIĆ, S.S., GRGUR, B.N., Fundamentals aspects of electrometallurgy, $1^{\text {a }}$ ed., New York, Kluwer Academic Publishers, 2002.

[3] BARD, A.J., FAULKNER, L.R., Electrochemical methods. Fundamentals and applications, $1^{\text {a }}$ ed., New York, John Wiley \& Sons Inc., 2001.

[4] PEREZ, N., Electrochemistry and corrosion science, $1^{\text {a }}$ ed., New York, Kluwer Academic Publishers, 2004.

[5] POMBO, F.R., DUTRA, A.J.B., "Eletrorrecuperação de cobre e oxidação de cianeto de efluentes cianídricos diluídos gerados por unidade de galvanoplastia", Matéria (Rio J.), v.13, n. 3, pp. 418-428, Jul. 2008.

[6] MIRZA, A., BURR, M., ELLIS, T., et al., "Corrosion of lead anodes in base metals electrowinning", The Journal of The Southern African Institute of Mining and Metallurgy, v. 116, n. 1, pp. 533-538, Jun. 2016.

[7] GUZMAN, D., GARCIA, C., SOLIZ, A., et al., "Synthesis and electrochemical properties of Ti-Si alloys prepared by mechanical alloying and heat treatment", Metals, v. 8, n. 6, pp. 417-429, Jun. 2018.

[8] ZHANG, W., GHALI, E., HOULZCHI, G., "Review of oxide coated catalytic titanium anodes performance for metal electrowinning", Hydrometallurgy, v. 169, n. 1, pp. 456-467, May. 2017.

[9] ZHANG, Y., GUO, Z., "Anodic behavior and microstructure of $\mathrm{Pb}-\mathrm{Ca}-0.6 \% \mathrm{Sn}, \mathrm{Pb}_{-} \mathrm{Co}_{3} \mathrm{O}_{4}$ and $\mathrm{Pb}-\mathrm{WC}$ composite anodes during $\mathrm{Cu}$ electrowinning", Journal of Alloys and Compounds, v. 724, n. 1, pp. 103-111, Nov. 2017.

[10] PAVLOVIĆ, M.G., PAVLOVIĆ, L.J., MAKSIMOVIĆ, V.M., et al., "Characterization and morphology of copper powder particles as a function of different electrolytic regimes", International Journal of Electrochemical Science, v. 5, n. 1, pp. 1862-1878, Dec. 2010.

[11] WAHYUDI, S., SOEPRIYANTO, S., MUBAROK, M.Z., et al., "Effect of pulse parameters on the particle size of copper powder electrodeposition", In: International Conference on Design and Applications of Engineering Materials, 012020, Bandung, Indonesia, 6-7 September 2018.

[12] YUE, S.X., SU, Y.C., LUO, Z.B., et al., "Influence of surfactant interaction on ultrafine copper powder electrodeposition", Materialwissenschaft und Werkstofftechnik, v. 50, n. 7, pp. 856-863, Jul. 2019.

[13] EHSANI, A., YAZICI, E.Y., DEVECI, H., "Influence of polyoxometallates as additive on electrowinning of copper", Hydrometallurgy, v. 162, n. 1, pp. 79-85, Jun. 2016.

[14] NAJMINOORI, M., MOHENNI, A., AFROOZ, K., et al., "The effect of magnetic field and operating parameters on cathodic copper winning in electrowinning process", Chemical Engineering Science, v. 199, n. 1, pp. 1-19, May. 2019.

[15] CHRZANOWSKA, A., MROCZKA, R., "Influence of chloride anions and polyethylene glycol on the morphology of electrodeposited copper layers", Electrochimica Acta, v. 78, n. 1, pp. 316-323, Sep. 2012.

[16] DUTRA, A.J.B., ROCHA, G.P., POMBO, F.R., "Recuperação de cobre eletrolítico a partir de solução gasta de cubas de eletrodeposição em meio cianídrico", Matéria (Rio J.), v. 12, n. 1, pp. 237-244, Mar. 2007.

[17] SILVA, P.S., LAGO, D.C.B., SENNA, L.F., "Produção e caracterização de revestimentos de ligas metálicas Cu-Sn em banho eletrolítico contendo glicina: ensaios preliminares", Matéria (Rio J.), v. 24, n. 3, Jan. 2019.

[18] OLIVEIRA, J.A.M., RAULINO, A.M.D., RAULINO, J.L.C., et al., "Efeito da densidade de corrente e pH na obtenção da liga Ni-Fe por eletrodeposição", Matéria (Rio J.), v. 22, n. 1, e11773, Abr. 2017.

[19] NIKOLIĆ, N.D., AVRAMOVIĆ, L., IVANOVIĆ, E.R., et al., "Comparative morphological and crystallographic analysis of copper powders obtained under different electrolysis conditions", Transactions of Nonferrous Metals Society of China, v. 29, n. 6, pp. 1275-1284, Jul. 2019.

[20] ASLAN, E., PATIR, I.H., ERSOZ, M., "Cu nanoparticles electrodeposited at liquid-liquid interfaces: A highly efficient catalyst for the hydrogen evolution reaction", Chemistry - A European Journal, v. 21, n. 1, pp. 4585-4589, Mar. 2015.

[21] WANG, Z., CAI, X., YANG, C., et al., "An electrodeposition approach to obtaining carbon nanotubes embedded copper powders for the synthesis of copper matrix composites", Journal of Alloys and Compounds, v. 735, n. 1, pp. 1357-1362, Feb. 2018.

[22] DUTRA, A.J.B., ALMEIDA, I.C.F., "Efeito da densidade de corrente e temperatura na área superficial do dióxido de manganês eletrolítico", Matéria (Rio J.), v. 11, n. 1, pp. 60-69, Mar. 2006. 
[23] MROCZKA, R., ŁOPUCKI, R., ŻUKOCIŃSKI, G., "Molecular analysis of additives and impurities accumulated on copper electrodeposited layer by time-of-flight secondary ion mass spectrometry", Applied Surface Science, v. 463, n. 1, pp. 412-426, Jan. 2019.

[24] SHAO, W., PATTANAIK, G., ZANGARI, G., "Influence of chloride anions on the mechanism of copper electrodeposition from acidic sulfate electrolytes", Journal of The Electrochemical Society, v. 154, n. 4, pp. D201-D207, Feb. 2007.

[25] POURBAIX, M.J.N., Thermodynamics of dilute aqueous solutions, $1^{\mathrm{a}}$ ed., London, Edward Arnold \& Co, 1949.

[26] BOCKRIS, J.O.M., RAZUMNEY, G.A., Fundamental aspects of electrocrystallization, $1^{\text {a }}$ ed., New York, Plenum Press, 1967.

\section{ORCID}

Alvaro David Soliz Ayala

Catalina Natalia Alfaro Gallardo

Luis Reynaldo Cáceres Villanueva

Danny Guzman Mendez
https://orcid.org/0000-0002-6548-956X

Sin información

https://orcid.org/0000-0001-7359-2148

https://orcid.org/0000-0002-8020-9247 